\title{
Aqueous Colloidal Stability of Graphene Oxide and Chemically Converted Graphene
}

\author{
Swarnima Kashyap, ${ }^{1}$ Shashank Mishra, ${ }^{2}$ and Shantanu K. Behera' \\ ${ }^{1}$ Department of Ceramic Engineering, National Institute of Technology Rourkela, Odisha 769 008, India \\ ${ }^{2}$ Department of Ceramic Engineering, Indian Institute of Technology BHU, Varanasi, Uttar Pradesh 221 005, India
}

Correspondence should be addressed to Shantanu K. Behera; behera@alum.lehigh.edu

Received 6 August 2014; Revised 24 October 2014; Accepted 24 October 2014; Published 13 November 2014

Academic Editor: Vijaya Rangari

Copyright (c) 2014 Swarnima Kashyap et al. This is an open access article distributed under the Creative Commons Attribution License, which permits unrestricted use, distribution, and reproduction in any medium, provided the original work is properly cited.

Graphene oxide (GO) was prepared by modified Hummer's method, and chemically converted graphene (CCG) was prepared by further reduction of the aqueous $\mathrm{GO}$ colloid. The effect of $\mathrm{pH}$ on particle size, particle charge, and light absorption of the aqueous colloids of GO and CCG was studied with titration against $\mathrm{HCl}$ or $\mathrm{NaOH}$, to find the ideal characteristics for a stable dispersion. The GO colloid was stable in the $\mathrm{pH}$ range of 4-11, whereas the CCG colloid gained stability at a relatively narrower $\mathrm{pH}$ range of 7-10. Poor stability of the colloids was observed for both GO and CCG colloids at both extremes of the pH scale. Both of the colloids exhibited average size of $\sim 1$ micron in the low $\mathrm{pH}$ range, whereas for higher $\mathrm{pH}$ the size ranged between 300 and $500 \mathrm{~nm}$. The UV-Vis spectra showed absorption peak at $230 \mathrm{~nm}$ for GO colloids that shifted to $260 \mathrm{~nm}$ for the CCG colloid. Such shift can be ascribed to restoring of electronic conjugation of the $\mathrm{C}=\mathrm{C}$ bonds in $\mathrm{CCG}$.

\section{Introduction}

Graphene is a flat 2D layer of carbon atoms packed in a honeycomb lattice and is the basal building block in all graphitic materials [1]. Its different synthesis routes and the possession of unique mechanical, thermal, and electrical properties make it a material of great interest [2-7]. In spite of the viable structure-property relationship, large volume production of graphene is still a challenge. Graphene oxide syntheses via chemical methods using acids and oxidants are the most widely followed procedures, which can be further reduced to produce graphene [8-10]. Graphene oxide (GO) can be best described as a single layer planar hexagonal array of carbon atoms to which functional groups, including carboxylic acid, hydroxyl, epoxy, and carbonyl groups, are attached. According to the Lerf-Klinowski model, the carbonyl and carboxylic groups remain attached to the edges of the sheet, while the hydroxyl and epoxy groups are found on the basal plane [11]. These groups render the GO sheets hydrophilic due to the H-bonding present between the carboxylic and hydroxyl groups. This property opens up many opportunities for chemical reactions and schemes, including self-assembly and thin films processing, to exploit the beneficial properties of graphene oxide. On the contrary, graphene sheets that are devoid of any $\mathrm{O}$-containing functional groups are insoluble in water, thus posing a challenge for the exploitation of graphene in aqueous processing methods. Several methods, including functionalization [12-14], in situ reduction $[15,16]$, or polymeric reduction [17] of GO sheets in aqueous medium, can be used to obtain graphene based colloids. Therefore, to afford graphene based nanostructures, the foremost task is to develop aqueous dispersions of graphene sheets by chemical reduction of GO sheets, generally termed as chemically converted graphene (CCG).

The behaviour of graphene oxide colloids is influenced by various parameters, including $\mathrm{pH}$, particle size, and zeta potential. An understanding of these parameters and their influence on the stability of the colloids is key to exploiting aqueous synthesis routes for the fabrication of graphene based hybrids and composites. In this work we report the change in zeta potential, light absorption, and equivalent particle size of graphene oxide and chemically converted 
graphene colloids over a range of $\mathrm{pH}$. Both CCG and GO sheets are negatively charged colloidal particles carrying varying concentration of functional groups. Oxygencontaining functional groups present on the graphene oxide sheets make GO highly hydrophilic. On the other hand, CCG, or partially reduced colloid of graphene oxide, uses the remnant oxygen-containing groups for displaying the stability of mildly oxidised graphene oxide sheets. As the colloid is titrated with $\mathrm{NaOH} / \mathrm{HCl}$, the ensuing change in the $\mathrm{pH}$ of the colloid leads to agglomeration of the graphene based colloidal particles. With very few reports elucidating the individual behaviour of CCG or GO colloids over size, stability, or conjugation studies $[18,19]$, a comparative study of both of the colloids on the said parameters has not been reported. In the current work, the effect of $\mathrm{pH}$ on the zeta potential, optical absorption, and equivalent particle size of graphene oxide and chemically converted graphene colloids is reported.

\section{Experimental Details}

Flake graphite (98\%), sulphuric acid (98\%), hydrochloric acid (37\%), hydrogen peroxide (30 wt\%), potassium permanganate, hydrazine monohydrate ( $98 \mathrm{wt} \%$ in water), ammonia (35\%), and sodium hydroxide were obtained from commercial sources and used without any further purification. Graphene oxide was prepared by modified Hummer's method as described by Chen et al. [20]. Graphite powder $(2 \mathrm{~g})$ was poured into concentrated $\mathrm{H}_{2} \mathrm{SO}_{4}(46 \mathrm{~mL})$ at $0^{\circ} \mathrm{C}$ and stirred for about $30 \mathrm{~min}$. Thereafter, $\mathrm{KMnO}_{4}(6 \mathrm{~g})$ was added gradually while stirring the batch for about 30 minutes. Continuous cooling was ensured so that the temperature of the mixture does not exceed $20^{\circ} \mathrm{C}$. The reaction between $\mathrm{H}_{2} \mathrm{SO}_{4}$ and $\mathrm{KMnO}_{4}$ leads to the formation of metal and sulphate ions that help in exfoliating the graphite layers [21]. The mixture was then heated up to $35^{\circ} \mathrm{C}$ and was kept for more than $30 \mathrm{~min}$. Deionized water $(92 \mathrm{~mL})$ was gradually added. After $60 \mathrm{~min}$ of further stirring, the reaction was terminated by the addition of a large amount of deionized water and $30 \% \mathrm{H}_{2} \mathrm{O}_{2}$ solution $(5 \mathrm{~mL})$. This caused violent effervescence and an increase in temperature (up to $100^{\circ} \mathrm{C}$ ), after which the color of the suspension changed to bright yellow. The suspension was then washed with $1: 10 \mathrm{HCl}$ solution $(5 \mathrm{~L})$ in order to remove metal ions by filter paper and funnel. The paste collected from the filter paper is dried at $60^{\circ} \mathrm{C}$ until it became agglomerated. This agglomerate was dispersed into deionized water in static state for $2-3 \mathrm{~h}$ and slightly stirred by a glass rod. The suspension was washed with more deionized water several times until the $\mathrm{pH}$ reached a value of 7 . The collected paste was ultrasonicated for about $1 \mathrm{~h}$ for better dispersion in water. The obtained brown GO hydrosol was then centrifuged at $4000 \mathrm{rpm}$ for $30 \mathrm{~min}$ in order to remove any unexfoliated GO. Scanning electron microscopy of a drop of the colloid (after drying) exhibited crumpled graphene oxide sheets.

Chemically converted graphene (CCG) was prepared as per the process described by $\mathrm{Li}$ et al. [22]. In short, $5 \mathrm{mg}$ graphene oxide powder was dispersed in $100 \mathrm{~mL}$ of distilled water by ultrasonication until a yellowish-brown colloid was obtained. The GO colloid was then transferred to a $250 \mathrm{~mL}$ round bottom flask and stirred for a few minutes. Subsequently, few drops of ammonia solution (35\%) were added to increase the $\mathrm{pH}$ up to 8 allowing stability of the sheets. $5 \mu \mathrm{L}$ hydrazine hydrate $(0.1 \mathrm{M})$ solution was added to the above solution and refluxed at $98^{\circ} \mathrm{C}$ for 1 hour in a water bath. The process afforded a black colloidal dispersion, hereafter called CCG colloid. The GO colloid and the CCG colloid were filtered and dried separately for additional characterizations.

The as-synthesized GO powder $(0.2 \mathrm{mg} / \mathrm{mL})$ was suspended in distilled water by bath sonication for 30 minutes, affording the formation of a uniformly dispersed yellowishbrown colloid. The stable colloid had $\mathrm{pH}$ of around 6.7 indicating that the $\mathrm{GO}$ sheets bore strong - $\mathrm{OH}$ bond affinity with the water molecules. At this stage, it can be assumed that the GO colloid was devoid of any metallic impurity or salt. The colloid was then divided into two halves. One half of the GO colloid was titrated with $0.1 \mathrm{M} \mathrm{HCl}$ solution to progressively decrease the $\mathrm{pH}$ from 6.7 to 1 . The second half was titrated with $0.1 \mathrm{M} \mathrm{NaOH}$ solution to increase the $\mathrm{pH}$ from 6.7 to 13. Every integral change in the $\mathrm{pH}$ of GO colloid was recorded by a $\mathrm{pH}$ meter (Mettler Toledo) and collected in $5 \mathrm{~mL}$ vials. GO colloids with $\mathrm{pH}$ ranging from 1 to 12 were obtained for further characterization. Similar procedures were followed to obtain CCG colloids with $\mathrm{pH}$ from 1 to 12 . To ensure a better dispersion, the samples were sonicated for 15 minutes before each characterization.

Size and morphology of the GO powders were measured by scanning electron microscopy (JEOL XL 30). Surface charge of the colloidal dispersion (GO and CCG) was measured by zeta potential meter (Zetasizer, Malvern, UK) between the $\mathrm{pH}$ ranges of $2-12$, and the average particle size of the colloidal particles was measured by dynamic light scattering technique. UV-Vis absorption spectra were recorded by a spectrometer (Lambda 45, Perkin Elmer) within the wavelength range of $200-900 \mathrm{~nm}$.

\section{Results and Discussion}

$\mathrm{X}$-ray diffraction (XRD) of the graphite precursors, GO, and CCG was carried out over the range of 5 to $50^{\circ}$ of $2 \Theta$ with a scan rate of $2^{\circ} \mathrm{min}^{-1}$ (cf. Figure 1). The XRD pattern of graphite exhibited the characteristic peak at $26^{\circ}$ corresponding to (200) reflections. The XRD pattern for GO showed a peak at $\sim 11^{\circ}$, corresponding to an expanded interlayer distance of $7.6 \AA$. This is due to the intercalation of acidic functional groups in between the graphitic layers that causes an increase in the interplanar distance. In such a case, the (200) graphite peak shifts to lower $2 \Theta$ region in the vicinity of $10^{\circ}$. However, if the graphene layers are fully expanded and exfoliated to single layers, such a peak may not be observed for graphene oxide. In addition, the CCG powders (after filtration and drying) do not seem to restack, which is evident by the absence of peaks for CCG in the X-ray diffractogram (Figure 1). Single drops of the as-prepared GO colloid and CCG colloid (at $\mathrm{pH} \sim 7$ ) were cast on metallic stubs, and scanning electron microscopy was performed. Figure 2(a) shows a representative image of the GO sheets. Similarly, the morphology of CCG is shown in 


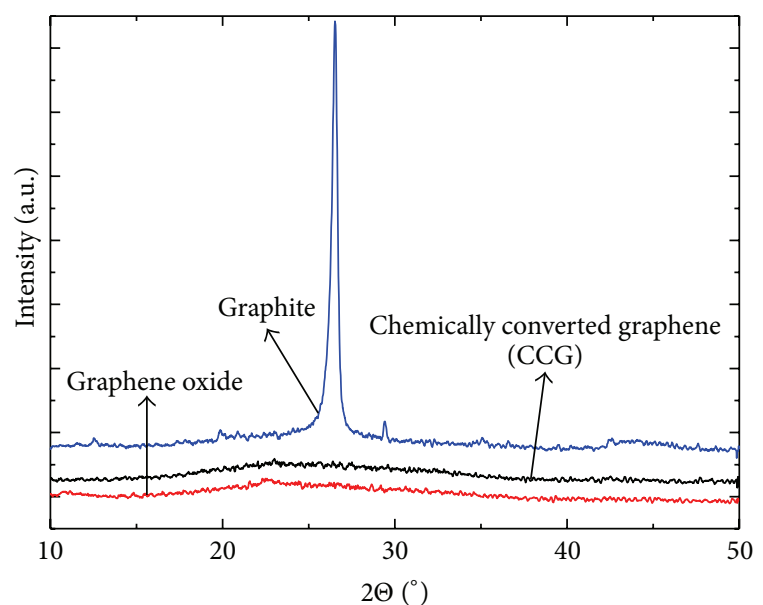

FIGURE 1: X-ray diffraction patterns of graphite, graphene oxide, and chemically converted graphene.

Figure 2(b). Subtle charging effects are seen from the SEM images because the samples were not coated. Moreover, it may be noted that we have taken the images (purposefully) at $20 \mathrm{kV}$, which is an exceedingly high voltage regime to image carbon based materials. This high voltage and the sharp edges of CCG exhibit subtle charging effects (chemically converted graphene cannot have as high electrical conductivity as that of pristine graphene; it may lie somewhere between that of graphene oxide and graphene).

The zeta potential $(\zeta)$ is an important factor for characterizing the stability of colloidal dispersions. It is a measure of the negative charge around the double layer associated with the colloidal particle as a consequence of the ionization of different functional groups. Generally, particles with zeta potential in the range $-30 \mathrm{mV}$ to $+30 \mathrm{mV}$ are considered stable due to electrostatic repulsion [23]. Our measurements show that zeta potential for the GO colloids is $\mathrm{pH}$ sensitive, and effective dispersion of the colloids occurred within the pH range of 4-12 (cf. Figure 3). Experimentally, we observed that and the highest magnitude of $\zeta$ is obtained at $\mathrm{pH} 10$ $(-48.6 \mathrm{mV})$, although the $\mathrm{pH}$ range of $7-11$ showed the highest (negative) charge.

Dynamic light scattering (DLS) results for both GO and CCG colloids showed similar trends with subtle differences. It must be mentioned here that we do not attempt to estimate the average particle size of GO and CCG. DLS is a technique that is well suited for estimating the size of spherical shaped particles. GO and CCG colloids, on the contrary, are particulate systems that possess extremely large ratios of length or breadth (few microns) to thickness (few nanometers). Therefore, the DLS pattern of such a system could provide a convoluted result, which is expected to be close to the lateral dimensions of the GO and CCG platelets. However, the objective here is to compare the relative change in the platelet size as a function of $\mathrm{pH}$. For the graphene oxide sample at $\mathrm{pH} 7$, the size distribution varied in the range of $250 \mathrm{~nm}$ to $570 \mathrm{~nm}$ (cf. Figure 4). But, as the sample was titrated with $\mathrm{NaOH}$ to a higher $\mathrm{pH}$, the population lays in the range of approximately $300 \mathrm{~nm}$ to $500 \mathrm{~nm}$. The stability of the
GO colloid improves as $\mathrm{NaOH}$ acts as a hydrogenating agent for graphene oxide [24]. Moreover, it has also been reported that $\mathrm{NaOH}$ has an ability to successfully remove the oxygen functionalities from the surface of graphene oxide to form activated graphene sheets $[25,26]$. These activated sheets begin to stabilize themselves in the solution by reducing their effective size. Thus, the change in color and stability of the $\mathrm{NaOH}$ titrated samples could be due to the development of small sized activated graphene sheets. GO colloids, on the contrary, when titrated with $\mathrm{HCl}$ result in very large sheet size and poor stability. This can be attributed to the increase in $\mathrm{H}^{+}$ ions in the solution, which gradually increase the sheet size and reduced stability.

These observations reiterate our findings from the zeta potential measurements that GO colloids are more stable in the 3-11 pH range. The thickness of the GO sheets is normally expected to increase with decrease in $\mathrm{pH}$ due to increasing protonation of acidic groups $(\mathrm{C}-\mathrm{OH}, \mathrm{COOH})$ present on the sheets. Protonation decreases the negative charge on the sheets, thereby decreasing electrostatic repulsion between them, eventually resulting in the agglomeration of the sheets and reduced colloidal stability. However, we observe that, in the $\mathrm{pH}$ range of 6-9, the particle size distribution remains almost the same, and the distributions for colloids of $\mathrm{pH}$ 10 and 11 exhibit tails towards higher size. The reason for a slightly higher size distribution in $\mathrm{pH} 10$ and $\mathrm{pH} 11$ could be ascribed to the salting out effect of the $\mathrm{NaOH}$ additives. The CCG colloids, on the other hand, display sheets size in the range of $200 \mathrm{~nm}$ to $700 \mathrm{~nm}$ at $\mathrm{pH} 7$ (cf. Figure 5). On titrating them with $\mathrm{NaOH}$, the CCG sheets in the $\mathrm{pH}$ range from 7 to 10 stabilize uniformly and lie in the range of $200 \mathrm{~nm}$ to $400 \mathrm{~nm}$. But, above $\mathrm{pH} \mathrm{10}$, the sheets agglomerate and their size sharply increases over 1 micron, with very few sheets in the nanometric range (not included in Figure 5). This dramatic shift is supported by Fan et al. [27], who comment that the stability of the CCG sheets is attributed to a strengthened electrostatic stabilization under alkaline conditions, as the repulsion between the negatively charged sheets should increase at higher $\mathrm{pH}$ values. Thus, as the repulsion lies within their threshold limits, the CCG is stable, and above a threshold limit the sheets begin to agglomerate and destabilize themselves.

Overall, the DLS spectra of GO and CCG colloids exhibited size in the range of $200 \mathrm{~nm}$ to $1000 \mathrm{~nm}$, with the distribution tailing off at higher sizes for the $\mathrm{pH}$ range of 310. The distributions for colloids of $\mathrm{pH}$ 1-2 and $\mathrm{pH}$ 10-11 were completely different from the distributions in the $\mathrm{pH}$ range of 3-9, the average being observed beyond 1 micron at the $\mathrm{pH}$ extremes. This result closely matches with our common observation of a highly agglomerated GO colloid that is not transparent yellow but showed a muddy appearance.

UV spectroscopy was studied to observe the plasmon transitions for the titrated GO and CCG colloids. Graphene based colloids can exhibit interesting absorption patterns at the wavelength regions of $\sim 230 \mathrm{~nm}, \sim 260 \mathrm{~nm}$, and $\sim 300 \mathrm{~nm}$, corresponding to transitions involving $\pi, \sigma$, and $\mathrm{n}$ electrons of different bond energies. The titrated GO colloids produced a characteristic peak at $\sim 230 \mathrm{~nm}$ which is attributed to the $\pi-\pi^{*}$ plasmon of the $\mathrm{C}=\mathrm{C}$ bonds. This peak is similar for 


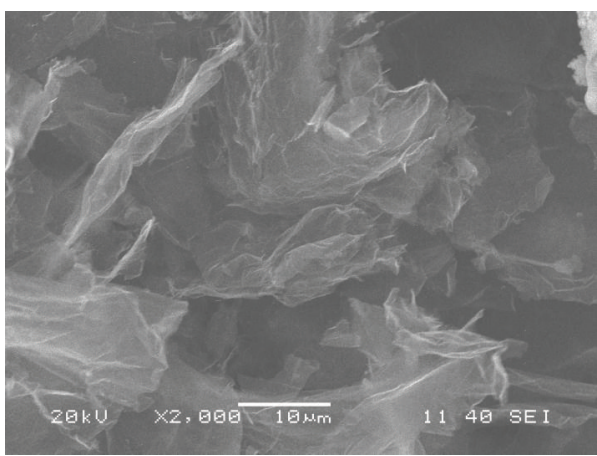

(a)

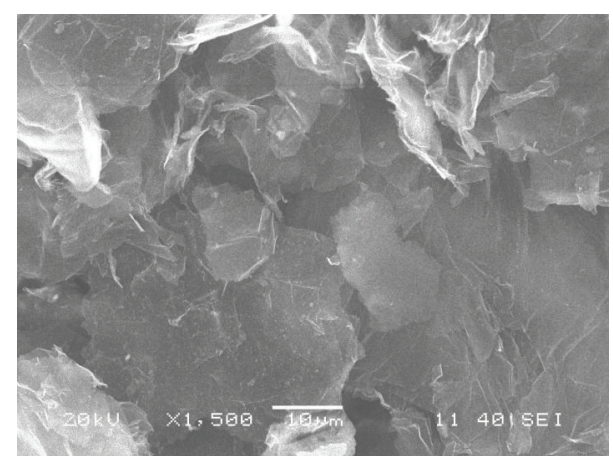

(b)

FIGURE 2: Scanning electron micrographs of (a) graphene oxide and (b) chemically converted graphene.

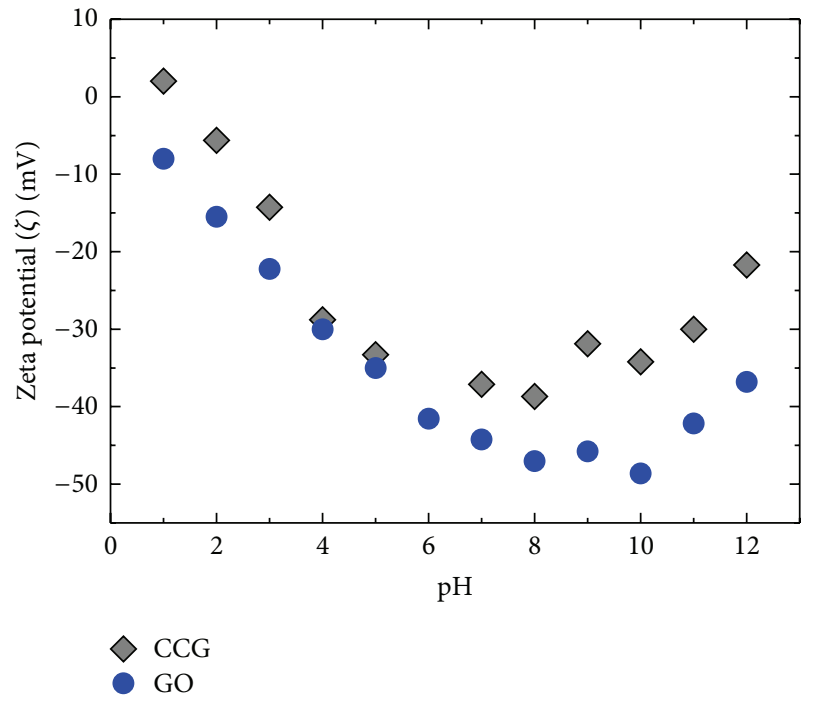

FIGURE 3: Zeta potential of GO and CCG colloids at different $\mathrm{pH}$.

samples in the $\mathrm{pH}$ range of 2 to 11 (cf. Figure 6). However, at $\mathrm{pH} 1$ and $\mathrm{pH} 12$, the absorption pattern appeared completely different, roughly indicating a continual decay of absorption with wavelength. At $\mathrm{pH}$, this arises as a result of high amount of sedimentation that takes place due to the aggregation and low repulsion within the sheets. As for $\mathrm{pH} \mathrm{12,} \mathrm{the}$ sedimentation occurs due to the salting out of the excess sodium ions. The UV-Vis spectra for the CCG colloids, on the other hand, displayed the plasmon peak at $260 \mathrm{~nm}$, which is a considerable peak shift to a higher wavelength (cf. Figure 7). This is due to the reduction of the $\mathrm{C}=\mathrm{O}$ groups and restoration of conjugation in the graphene sheets that lowers the energy required, thus increasing the wavelength of the $\pi-\pi^{*}$ transitions. This also predicts that CCG possesses substantially decreased oxygen content and higher sheet size for which the value of $\lambda_{\max }$ shifts to a higher wavelength. With the restoration of the conjugation in the hexagonal carbon sheets, the material is more graphene-like and is expected to show high thermal and electrical conductivity (also evident from the absorbing black color of the colloid).

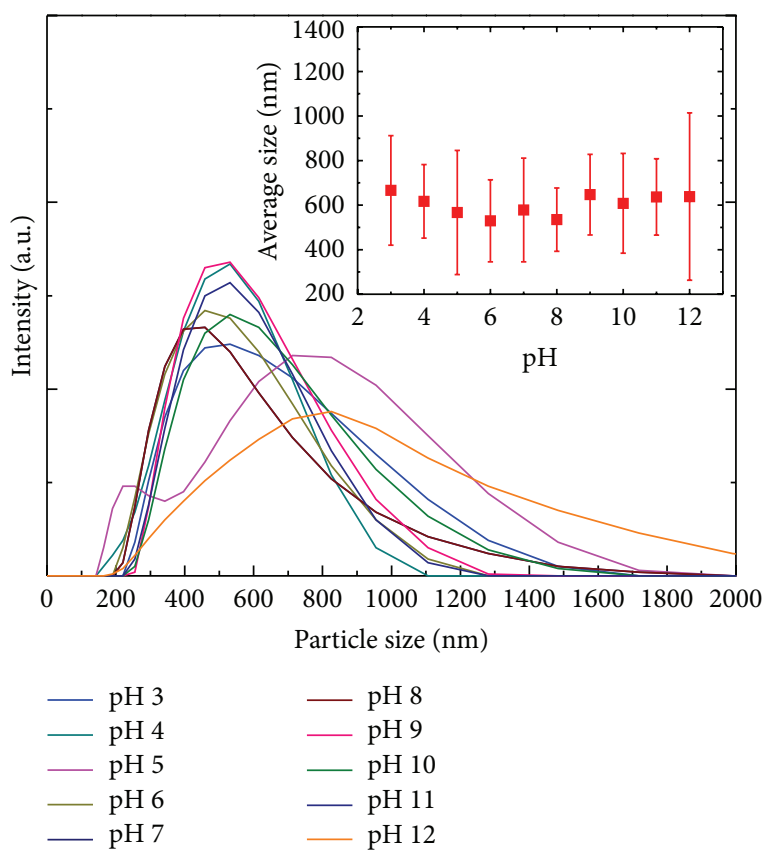

Figure 4: Particle size distribution of GO colloids at different $\mathrm{pH}$; inset shows the average particle size.

For the GO colloids, we also observed a shoulder peak at $300 \mathrm{~nm}$ (cf. Figure 6). Literature suggests that there exists a bond excitation value at $\sim 300 \mathrm{~nm}$ corresponding to the $\mathrm{n}-\pi^{*}$ transition of the $\mathrm{C}=\mathrm{O}$ chromophores for aldehyde and ketone molecule [28]. However, this peak can shift slightly due to the increased solvent polarity of graphene oxide as it is titrated with $\mathrm{NaOH} / \mathrm{HCl}$. On the other hand, this peak almost vanishes for the titrated CCG colloids indicating the absence of the $\mathrm{C}=\mathrm{O}$ chromophores in the colloid.

\section{Summary}

Aqueous dispersions of graphene oxide were found to be stable in the $\mathrm{pH}$ range of $4-12$, with excellent dispersibility in the range of 7-11, as evidenced by highly negative zeta potential. The colloidal stability of chemically converted 


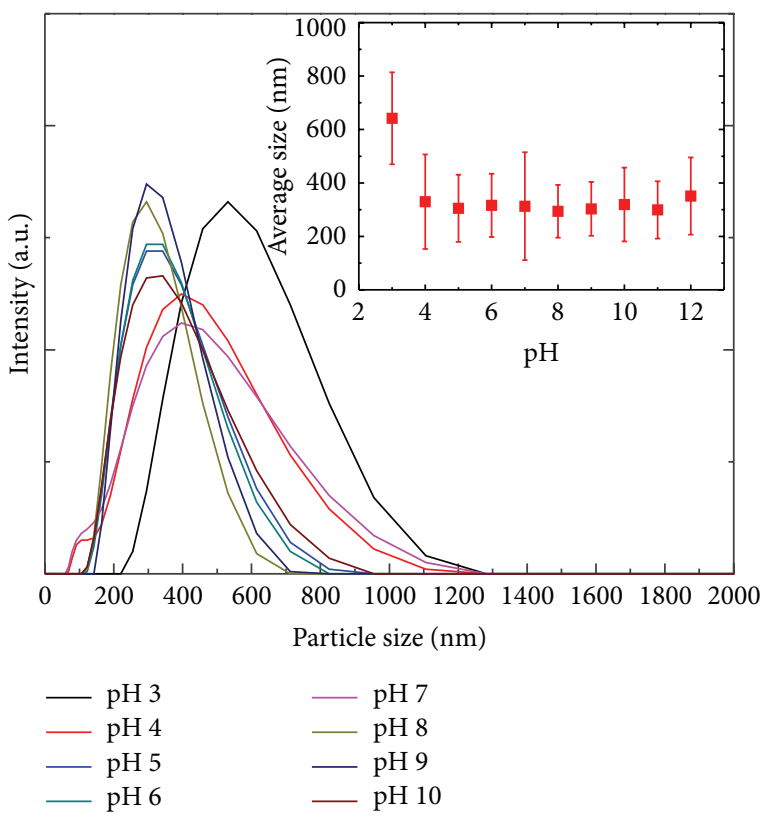

Figure 5: Particle size distribution of CCG colloids at different $\mathrm{pH}$; inset shows the average particle size.

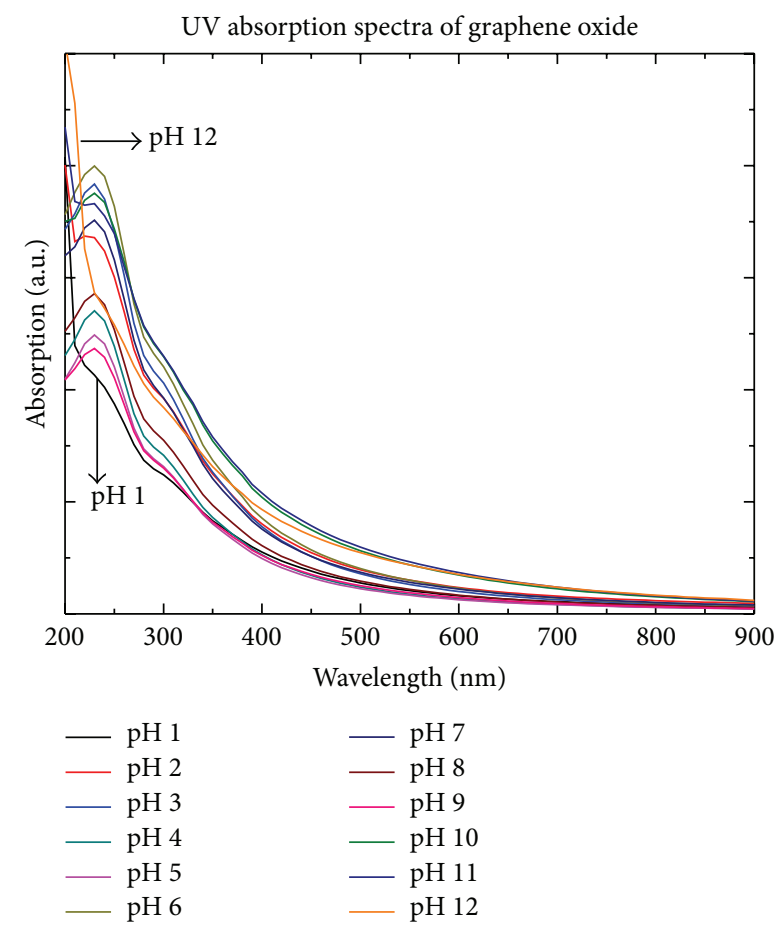

FIGURE 6: UV-Vis spectroscopy of GO colloids from acidic to basic conditions.

graphene in aqueous media was also found to be similar to that of graphene oxide colloids. UV-Vis spectroscopy of the chemically converted graphene colloids indicated the restoration of electronic conjugation in the graphene sheets. These findings are useful in the fabrication of graphene based composites and hybrids from aqueous processing methods.

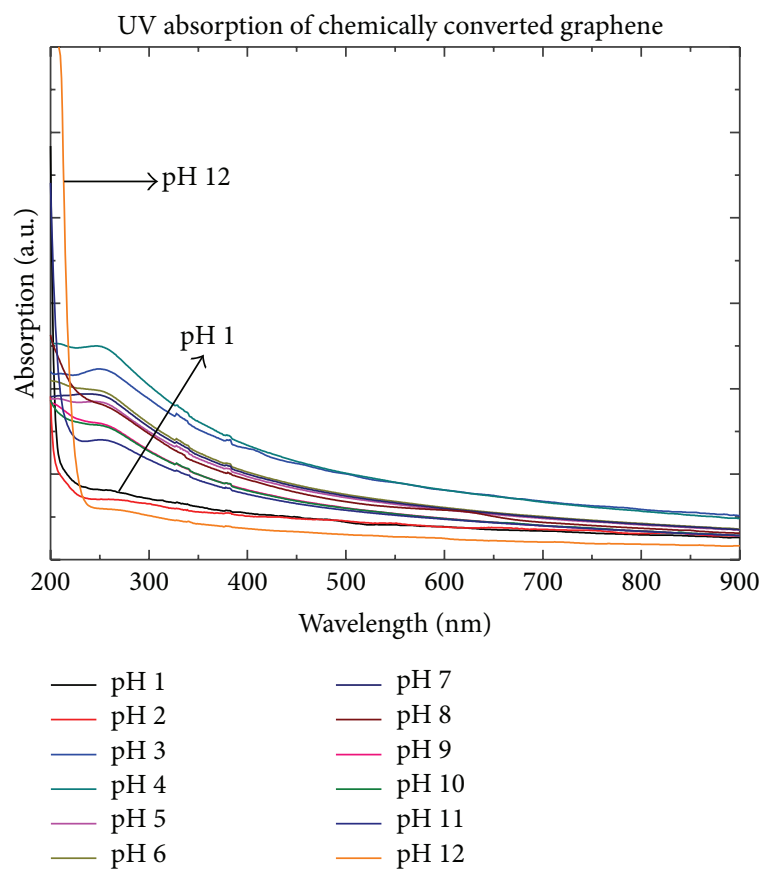

FIGURE 7: UV-Vis spectroscopy of CCG colloids from acidic to basic conditions.

\section{Conflict of Interests}

The authors declare that there is no conflict of interests regarding the publication of this paper.

\section{References}

[1] A. K. Geim and K. S. Novoselov, "The rise of graphene," Nature Materials, vol. 6, no. 3, pp. 183-191, 2007.

[2] K. S. Novoselov, A. K. Geim, S. V. Morozov et al., "Twodimensional gas of massless Dirac fermions in graphene," Nature, vol. 438, pp. 197-200, 2005.

[3] B. H. Hong, J. Y. Lee, T. Beetz, Y. Zhu, P. Kim, and K. S. Kim, "Quasi-continuous growth of ultralong carbon nanotube arrays," Journal of the American Chemical Society, vol. 127, no. 44, pp. 15336-15337, 2005.

[4] H. B. Heersche, P. Jarillo-Herrero, J. B. Oostinga, L. M. K. Vandersypen, and A. F. Morpurgo, "Electrical transport in high quality graphene pnp junctions," Nature, vol. 5, p. 446, 2007.

[5] Y. Kopelevich and P. Esquinazi, "Graphene physics in graphite," Advanced Materials, vol. 19, no. 24, pp. 4559-4563, 2007.

[6] G. M. Rutter, J. N. Crain, N. P. Guisinger, T. Li, P. N. First, and J. A. Stroscio, "Scattering and interference in epitaxial graphene," Science, vol. 317, no. 5835, pp. 219-222, 2007.

[7] A. A. Balandin, S. Ghosh, W. Bao et al., "Superior thermal conductivity of single-layer graphene," Nano Letters, vol. 8, no. 3, pp. 902-907, 2008.

[8] S. Stankovich, D. A. Dikin, G. H. B. Dommett et al., "Graphenebased composite materials," Nature, vol. 442, no. 7100, pp. 282286, 2006.

[9] S. Stankovich, D. A. Dikin, R. D. Piner et al., "Synthesis of graphene-based nanosheets via chemical reduction of exfoliated graphite oxide," Carbon, vol. 45, no. 7, pp. 1558-1565, 2007. 
[10] G. Eda and M. Chhowalla, "Chemically derived graphene oxide: towards large-area thin-film electronics and optoelectronics," Advanced Materials, vol. 22, no. 22, pp. 2392-2415, 2010.

[11] D. R. Dreyer, S. Park, C. W. Bielawski, and R. S. Ruoff, "The chemistry of graphene oxide," Chemical Society Reviews, vol. 39, no. 1, pp. 228-240, 2010.

[12] H. Kim, D. Lee, J. Kim, T.-I. Kim, and W. J. Kim, "Photothermally triggered cytosolic drug delivery via endosome disruption using a functionalized reduced graphene oxide," ACS Nano, vol. 7, no. 8, pp. 6735-6746, 2013.

[13] C. Wang, Y. Chen, K. Zhuo, and J. Wang, "Simultaneous reduction and surface functionalization of graphene oxide via an ionic liquid for electrochemical sensors," Chemical Communications, vol. 49, no. 32, pp. 3336-3338, 2013.

[14] O. C. Compton, D. A. Dikin, K. W. Putz, L. C. Brinson, and S. T. Nguyen, "Electrically conductive "alkylated" graphene paper via chemical reduction of amine-functionalized graphene oxide paper," Advanced Materials, vol. 22, no. 8, pp. 892-896, 2010.

[15] L. Lai, L. Chen, D. Zhan et al., "One-step synthesis of $\mathrm{NH}_{2}$-graphene from in situ graphene-oxide reduction and its improved electrochemical properties," Carbon, vol. 49, no. 10, pp. 3250-3257, 2011.

[16] S. Liu, J. Tian, L. Wang, and X. Sun, "A method for the production of reduced graphene oxide using benzylamine as a reducing and stabilizing agent and its subsequent decoration with $\mathrm{Ag}$ nanoparticles for enzymeless hydrogen peroxide detection," Carbon, vol. 49, no. 10, pp. 3158-3164, 2011.

[17] A. J. Glover, M. Cai, K. R. Overdeep, D. E. Kranbuehl, and H. C. Schniepp, "In situ reduction of graphene oxide in polymers," Macromolecules, vol. 44, no. 24, pp. 9821-9829, 2011.

[18] F. Li, Y. Bao, J. Chai, Q. Zhang, D. Han, and N. Li, "Synthesis and application of widely soluble graphene sheets," Langmuir, vol. 26, no. 14, pp. 12314-12320, 2010.

[19] S. Gurunathan, J. W. Han, A. Abdal Dayem, V. Eppakayala, and J.-H. Kim, "Oxidative stress-mediated antibacterial activity of graphene oxide and reduced graphene oxide in Pseudomonas aeruginosa," International Journal of Nanomedicine, vol. 7, pp. 5901-5914, 2012.

[20] T. Chen, B. Zeng, J. L. Liu et al., "High throughput exfoliation of graphene oxide from expanded graphite with assistance of strong oxidant in modified Hummers method," Journal of Physics: Conference Series, vol. 188, Article ID 012051, 2009.

[21] D. R. Dreyer, S. Park, C. W. Bielawski, and R. S. Ruoff, "The chemistry of graphene oxide," Chemical Society Reviews, vol. 39, no. 1, pp. 228-240, 2010.

[22] D. Li, M. B. Müller, S. Gilje, R. B. Kaner, and G. G. Wallace, "Processable aqueous dispersions of graphene nanosheets," Nature Nanotechnology, vol. 3, no. 2, pp. 101-105, 2008.

[23] R. J. Hunter, "Electro kinetics and zeta potential," in Foundations of Colloid Science, vol. 2, pp. 376-377, Oxford University Press, New York, NY, USA, 2001.

[24] T. Kuila, P. Khanra, N. H. Kim, J. K. Lim, and J. H. Lee, "Effects of sodium hydroxide on the yield and electrochemical performance of sulfonated poly(ether-ether-ketone) functionalized graphene," Journal of Materials Chemistry A, vol. 1, no. 32, pp. 9294-9302, 2013.

[25] Y. Zhu, S. Murali, M. D. Stoller et al., "Carbon-based supercapacitors produced by activation of graphene," Science, vol. 332, no. 6037, pp. 1537-1541, 2011.

[26] D. Zhang, X. Zhang, Y. Chen, C. Wang, and Y. Ma, "An environment-friendly route to synthesize reduced graphene oxide as a supercapacitor electrode material," Electrochimica Acta, vol. 69, pp. 364-370, 2012.

[27] X. Fan, W. Peng, Y. Li et al., "Deoxygenation of exfoliated graphite oxide under alkaline conditions: a green route to graphene preparation," Advanced Materials, vol. 20, no. 23, pp. 4490-4493, 2008.

[28] F. Yang, Y. Liu, L. Gao, and J. Sun, "pH-sensitive highly dispersed reduced graphene oxide solution using lysozyme via an in situ reduction method," The Journal of Physical Chemistry C, vol. 114, no. 50, pp. 22085-22091, 2010. 

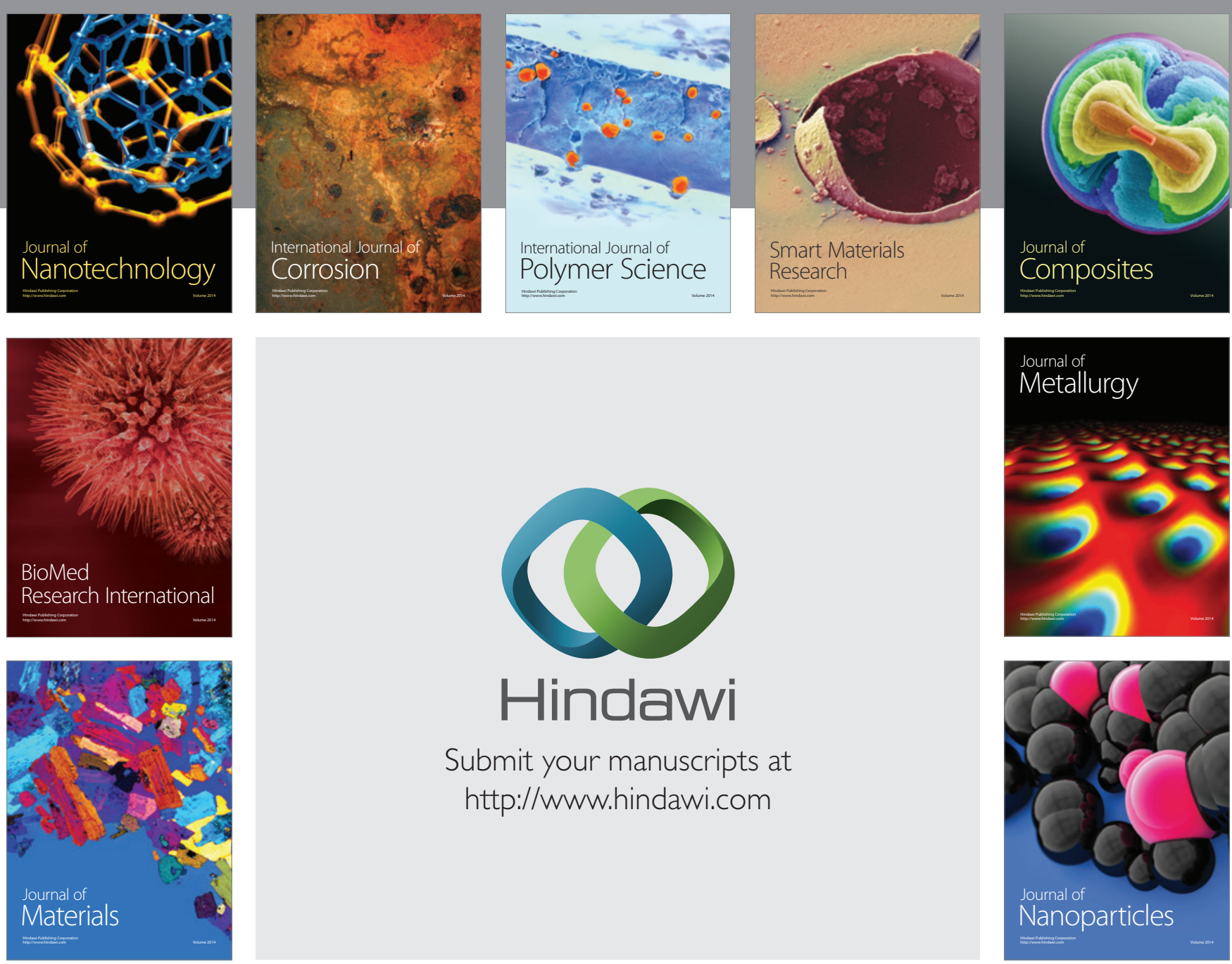

Submit your manuscripts at http://www.hindawi.com
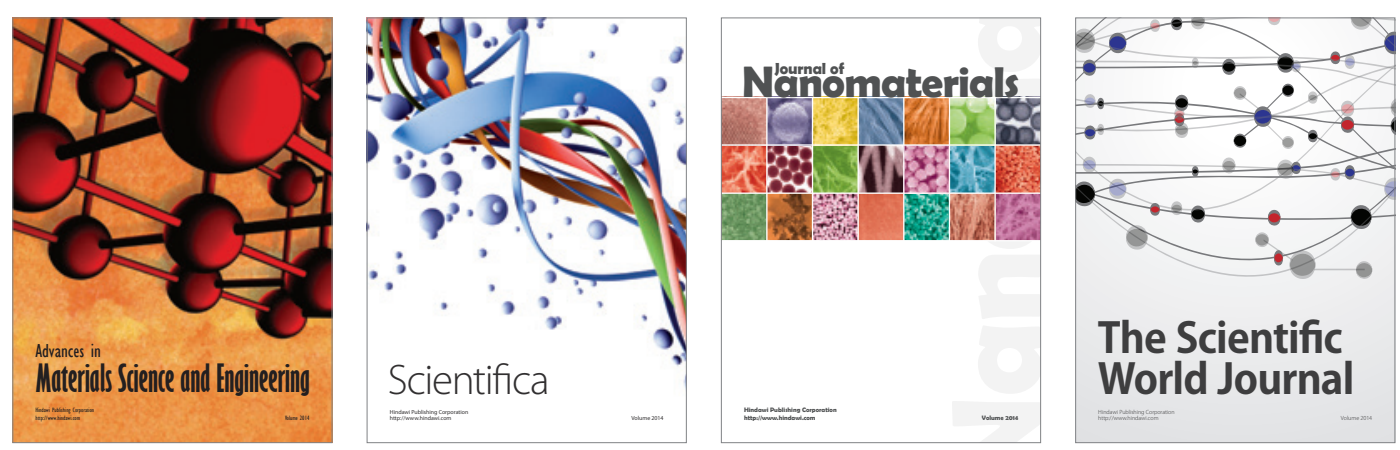

\section{The Scientific World Journal}
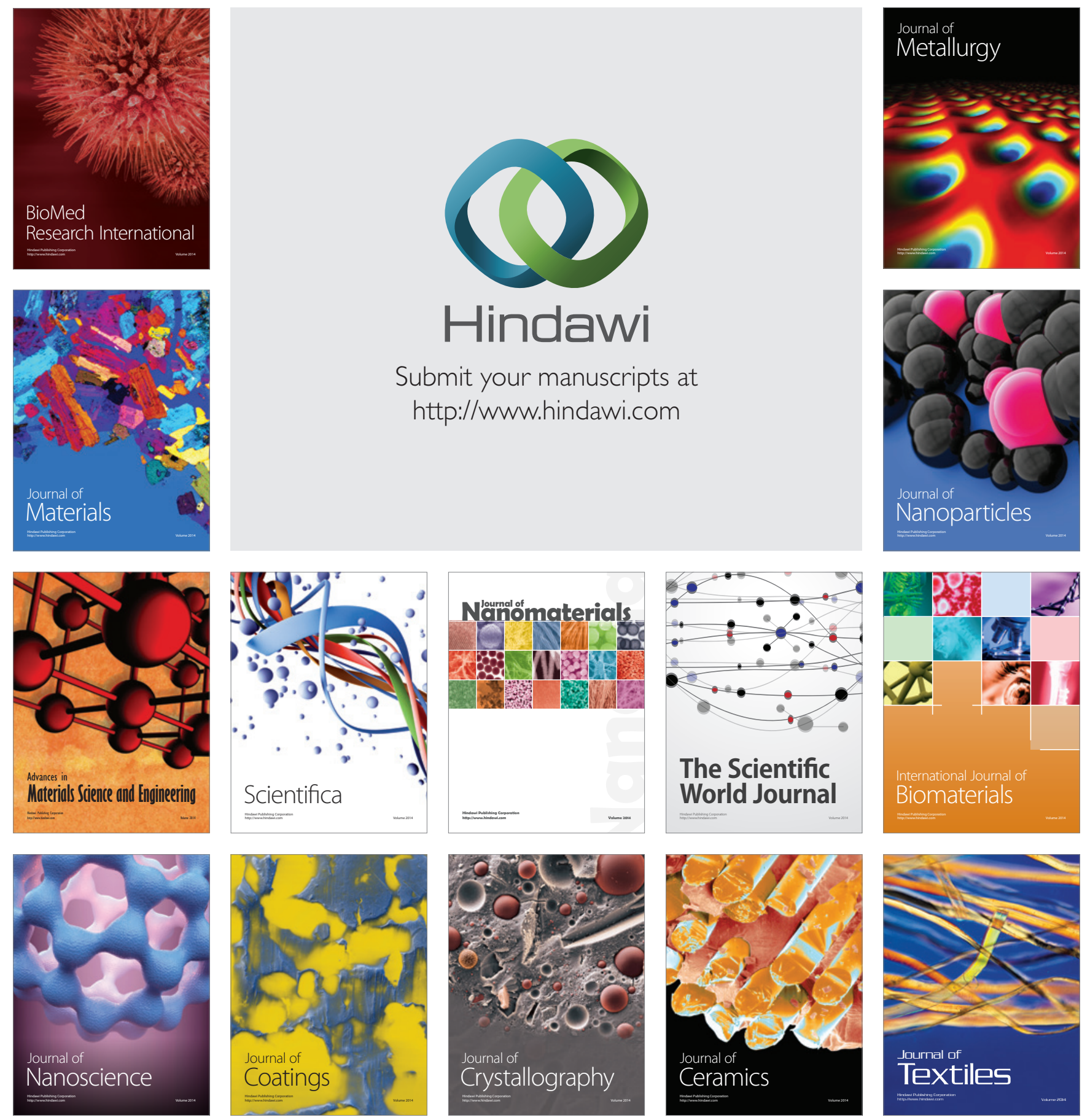